\title{
Realization and Evaluation of the Device for Measuring the Impedance of Human Body for Detecting the Respiratory and Heart Rate
}

\author{
Margus Metshein ${ }^{1}$, Toomas Parve ${ }^{1}$, Paul Annus ${ }^{1}$, Marek Rist ${ }^{1}$, Mart Min ${ }^{1}$ \\ ${ }^{I}$ Th. J. Seebeck Department of Electronics, Tallinn University of Technology, \\ Ehitajate tee 5, 19086 Tallinn, Estonia \\ margus.metshein@ttu.ee
}

\begin{abstract}
The idea of a device for measuring the impedance of human body with the target of monitoring the respiratory and heart rate is proposed in this paper. Hardware realization of the proposed idea is described with the illustration of the custom designed printed circuit board. Preparation of electrode shirts with various electrode placement configurations is introduced. Series of experimental measurements in the cases of dynamic bioimpedance reference and single human subject are described and results shown to evaluate the custom made device. The excitation frequencies in the range of $2 \mathrm{MHz}-$ $20 \mathrm{MHz}$ are utilized in the cases of large foil and textile electrodes to focus on the use of the capacitive connection to the object - constituting the novelty of the current paper. The results are analysed concerning the dependency of the visual availability of the interesting signal of breathing and heart rate of the material and the placement of the electrodes. Availability of breathing is found to be evident in all of the experimented cases. The heart rate is found to be challenging because of the presence of high frequency noise.
\end{abstract}

Index Terms-Capacitive electrode; electrical bioimpedance; synchronous detection; textile electrode.

\section{INTRODUCTION}

The monitoring of the vital signs has gained great interest in recent years - mainly because of the increasing availability of commercial smart monitoring devices and the wide spread usage of smartphones. The interest in personal health has grown through the self-awareness of the healthy lifestyle and the change of the daily movement habits in developed countries.

Several approaches are available for accessing to the respiratory rate $(\mathrm{RR})$ and heart rate (HR): piezo-resistive belt [1], different optical methods [2], inductive sensors [3] etc. A possibility of measuring the electrical bioimpedance (EBI) for tracking the changes of impedance of human body, caused by RR and HR, proposes an alternative.

There are number of papers, describing the ideas and realizations of wearable measurement devices, developed for monitoring the vital signs of human by using the EBI

Manuscript received 11 November, 2016; accepted 2 February, 2017.

This research was supported by Estonian Research Council grant IUT1911 and the European Regional Development Fund in frames of the Estonian Centre of Research Excellence EXCITE. The work was partly financed also through the European Research Area project H2020WIDESPREAD-2014-2-668995-Cognitive Electronics.
[4]-[6]. The approach of measuring the EBI by using the capacitive connection for monitoring the vital signs, is a product of recent years. The reasons are the concurrent problems of capacitive measurements, related to the affection of body movements in the form of motion artifact.

The motion artifact affects the results of the measurements of EBI, where the changes of impedance of the organism, caused by RR and HR, are relatively small. Currently, the influence of the motion artifact have not been considered and the measurements done in the cases where the object was sitting on the chair without moving.

The choice of the shape and the frequency of the excitation signal offers a point of decision in the case of the EBI measurements. Typically, the frequencies, proposed in different papers, stay below $1 \mathrm{MHz}$ [7] and sine wave is used. The square wave signal of frequencies in the range of $2 \mathrm{MHz}-20 \mathrm{MHz}$ are utilized in the current work despite the concurrent higher harmonics.

An impedance measurement device for detecting the RR and HR is presented in this paper. The basic idea and the realization of the device by using the custom designed printed circuit board is proposed. The results of the experimental measurements by using the prototype of the device in the cases of various large and small electrodes and electrode placements are shown and analysed.

\section{DEVICES AND PROPERTIES}

Number of laboratory devices were utilized in the experiments to implement the simulation models and support the experiments. For designing the printed circuit board (PCB) the Easily Applicable Graphical Layout Editor (EAGLE) of version 7.4.0 was used.

For powering the custom made measurement device, the Triple Output DC Power Supply of type E3631A, manufactured by Agilent Technologies, was used. Oscilloscope Infiniium DSO8104A, offering bandwidth of $1 \mathrm{GHz}$ and sampling rate of $4 \mathrm{GSa} / \mathrm{s}$, manufactured by Agilent Technologies, was utilized.

For the imitation of the changing impedance of human body, caused by RR and HR, the dynamic bioimpedance reference (DBR), designed at Tallinn University of Technology [8] was used.

The reference ECG was measured by custom-made 
measurement device in the case of using standard ECG solid gel electrodes according to the Eindhoven's lead III. A custom made spirometer with validated digital turbine flow meter was used for detecting the respiration.

As a reference device for measuring the impedance and its corresponding voltage level changes, HF2IS impedance spectroscope of Zurich Instruments with HF2TA transimpedance amplifier was used. The specification of these devices defines the frequency range up to $50 \mathrm{MHz}$ using either two- or four-electrode measurements [9]. Simultaneously, HF2IS was used to record the signals at the outputs of ECG measuring device and spirometer by using its auxiliary analog and digital signal inputs.

The gathering of the reference waveforms of HR and RR and processing of signals for gaining the spectrum was done by using corresponding Labview program.

\section{OVERVIEW OF THE CUSTOM MADE MEASUREMENT DEVICE FOR DETECTING THE RR AND HR}

\section{A. The Basic Idea and the Structure of the Device}

The idea of the device is to measure the impedance of the object by applying a certain signal, and measuring of the real part (ReŻ) of the total impedance by using two-electrode configuration [10]. In current paper, the calculation of the real part $(\operatorname{Re} \dot{Z})$ of the total impedance is not discussed as the proposal is given only for the analog portion of the circuit.

The device consists of 7 blocks (Fig. 1): excitation signal generator (1); inverting amplifier (2); synchronous detector (3); active signal filter (4); DC blocking high-pass filter (5); final stage amplifier (6); low-pass filter (7).

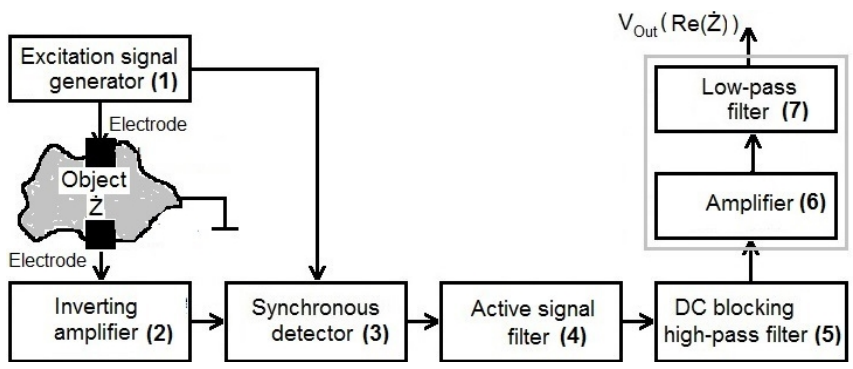

Fig. 1. Block diagram of the custom made measurement device.

The device was powered from bipolar power supply of $\pm 3 \mathrm{~V}$. At the output of the excitation signal generator, the square wave signal with adjustable frequency in the range of $0.5 \mathrm{MHz}-33 \mathrm{MHz}$, was available.

The gain of the inverting amplifier was set to 10 and the gain of the amplifier at the final stage was set to 220. The ratio of change of the voltage at the output of the fifth stage of the block diagram of the device stays in the order of $1 \mathrm{mV}$. The reason of the choice of the gain of the final stage amplifier is the desire of making the signals of RR and HR visible at the output of the device by using an oscilloscope.

The active signal filter is formed by using an AC op-amp integrator with DC gain control with the corner frequency of $15.92 \mathrm{kHz}$. This is used to smooth the waveform.

The high-pass filter was of $1^{\text {st }}$ order with the cut-off frequency of $1.05 \mathrm{~Hz}$ and the low-pass filter was of $2^{\text {nd }}$ order with the cut-off frequency of $1.94 \mathrm{~Hz}$. The reason for the choice of the bandwidth is the wish to recognize the HR and reduce the amplitude of $R R$, although $R R$ is still available.

The output of the device is low frequency voltage, induced by the in- and exhalation and the changing amount of blood in the object [10].

\section{B. The Hardware Realization of the Device}

In comparison to the first prototype of the impedance measurement device, described in [10], several changes were implemented. As an excitation signal generator, a special resistor set oscillator of type LTC1799CS5\#PBF, manufactured by Linear Technology, was used instead of logic gates. The frequency of the excitation signal was adjustable by changing the value of the frequency setting resistor.

A dual op-amp of type LT1813CS8\#PBF, manufactured by Linear Technology, was used for amplification instead of two discrete op-amps. LT1813CS8\#PBF offers the bandwidth of $100 \mathrm{MHz}$ and slew rate of $750 \mathrm{~V} / \mu \mathrm{s}$.

The device was realized by using custom designed PCB with surface-mount technology electronic components. The material of the PCB was FR-4 with the dimensions of $50 \mathrm{~mm} \times 80 \mathrm{~mm}$ and the thickness of $1.6 \mathrm{~mm}$. The PCB was designed as 2-sided board, where the whole bottom side was drawn as ground plane. The ground plane was drawn in two polygons, connected together with a line of width $0.41 \mathrm{~mm}-$ the reason is the intention to separate the ground of analog switch. The width of the signal lines on the PCB were set to $0.41 \mathrm{~mm}$ and for power lines to $0.61 \mathrm{~mm}$.

The layout of the designed PCB and the placement of the blocks can be seen in Fig. 2(a). The realized device can be seen in Fig. 2(b). E1 and E2 mark the connections for the wires of the electrodes.

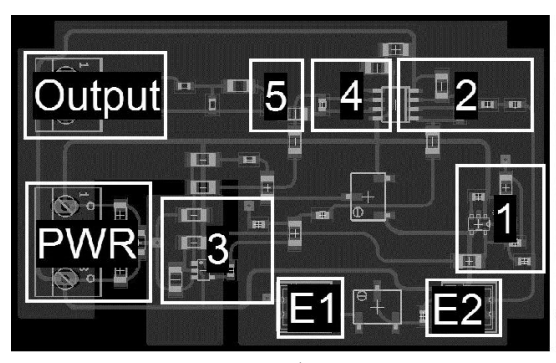

a)

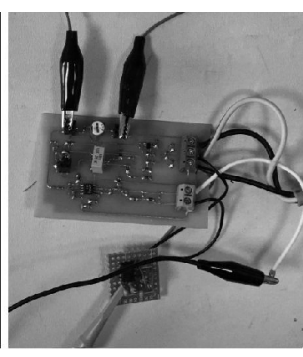

b)
Fig. 2. Layout of the designed PCB for the prepared measurement device.

The blocks of final stage amplifier and low pass filter (surrounded by grey line in Fig. 1) have not been added to the designed PCB because of the need for later implementations. These blocks were realized by using a small piece of prototyping $\mathrm{PCB}$, connected to the device with short wires. Op-amp TLV2782IP of Texas Instruments was used, offering the bandwidth of $8 \mathrm{MHz}$ with slew rate of $4.8 \mathrm{~V} / \mu \mathrm{s}$.

\section{Methods AND MEASUREMENT SETUP}

Measurement experiments were carried out to evaluate the capability of the implemented measurement device to follow the RR and HR in the cases of DBR and single volunteer (author of the current paper). 


\section{A. Measurement Setup in the Experiments by Using the $D B R$}

The DBR was used in the experiments - this device permits to adjust the parameters of the imitated impedance of human trunk separately, caused by both: the RR and HR [8]. For the measurements, the capacitive properties of the imitated RR and HR were disabled. The changes in the peakto-peak amplitudes of the resistances, caused by the RR and HR were set to $99.9 \Omega$ and $20 \Omega$ accordingly with the minimum value of $0 \Omega$. The frequencies of the RR and HR at the output of DBR have been set close to the same parameters of the human subject.

The electrode wires of the custom made measurement device were connected to the output of the DBR and the measurements done by using the direct electrical contact.

\section{B. Measurement Setup in the Experiments on Human by Using the Electrodes in Direct Connection}

Small monitoring electrodes with foam tape of type 2228 by $3 \mathrm{M}$ Health Care were used in the experiments. The electrodes were attached directly onto the skin in the predetermined positions (Fig. 3).

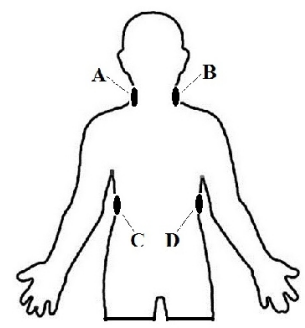

FRONT SIDE

Fig. 3. Positions of the electrodes in the case of direct contact measurements.

The positions were chosen to cover the trunk vertically so that the lungs and heart with the large blood vessels stay between the electrodes. The placement of the electrodes was inspired by [11], where, though, eight electrodes were used in four-electrode configuration.

\section{Measurement Setup in the Experiments on Human by} Using the Various Electrodes in Capacitive Connection

Three custom made electrode shirts with various electrode dimensions, materials and placements, were used in the experiments. Concerning all the shirts, the electrodes were used in noncontact setup, attached onto the outer surface of the shirt.

Electrode shirt no. 1 (ES1) included the large wet plate surface electrodes of original size that were positioned to cover the whole back, sides and shoulders of human (Fig. 4) [10], [12]. The placement was expected to grant access to the periodical changes that take place in lungs and heart.

Electrode shirt no. 2 (ES2) included the wet plate surface electrodes that were positioned onto the shoulders and upper arms (Fig. 5). The placement was expected to give access to the pulsating blood in subclavian arteries and veins.

The dimensions of the electrodes $\mathrm{J}$ and $\mathrm{K}$ were cut to $60 \mathrm{~mm} \times 80 \mathrm{~mm}$ and the dimensions of the electrodes $\mathrm{D}$ and E were cut to $80 \mathrm{~mm} \times 120 \mathrm{~mm}$. The electrodes were attached onto the outer surface of the cotton shirt by using the original foam tape of the electrodes

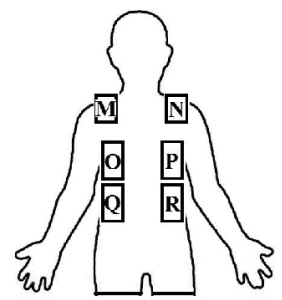

FRONT SIDE

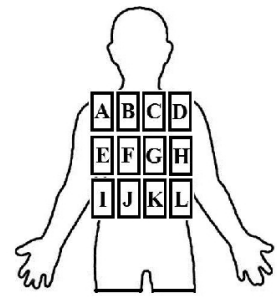

BACK SIDE

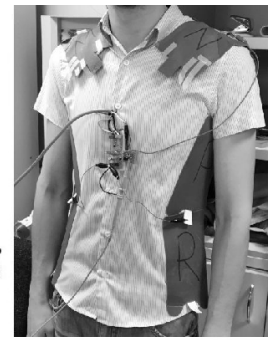

Fig. 4. Positions of the electrodes in the case of ES1 [5].

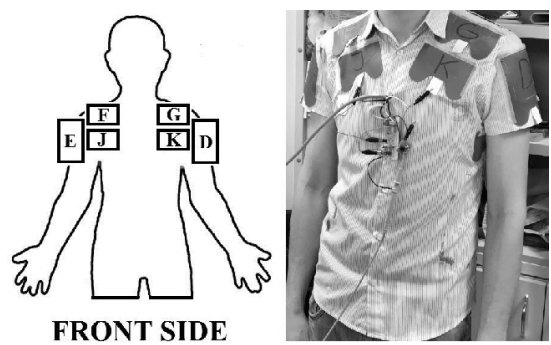

Fig. 5. Positions of the electrodes in the case of ES2

In the case of ES1 and ES2, the electrodes were connected together with wires to achieve the desired electrode placement configurations (EPC).

The electrodes, used in ES1 and ES2, were of type 22 4773 Electrosurgical Grounding Plates by Niko Medical Products with the contact surfaces of dimensions of $80 \mathrm{~mm} \times$ $170 \mathrm{~mm}$.

Electrode shirt no. 3 (ES3) included the conductive metallized nylon fabric (rip-stop) textile electrodes of part number 1500101130, manufactured by Statex Productions \& Vertriebs $\mathrm{GmbH}$, with dimensions of $80 \mathrm{~mm} \times 150 \mathrm{~mm}$. The chosen placement was directed to the use of two-electrode configuration (Fig. 6).

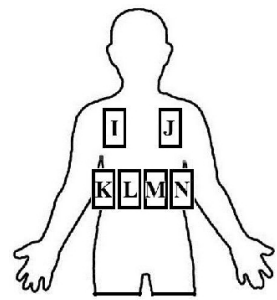

FRONT SIDE

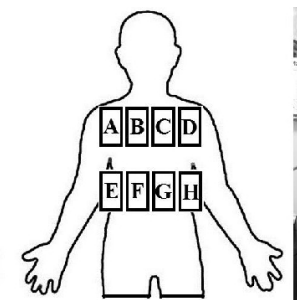

BACK SIDE

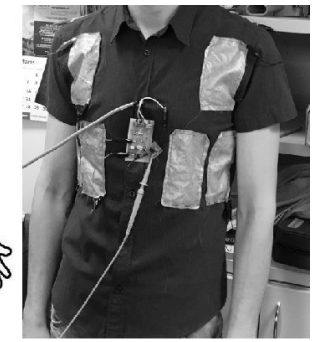

Fig. 6. Positions of the electrodes in the case of ES3.

The textile electrodes were connected by using the conductive thread of type Electro-Fashion Conductive Thread, manufactured by Kitronik, to constitute the desired EPC.

During the measurement experiments on human subject by using ES1-ES3, an additional cotton shirt with the thickness of $0.5 \mathrm{~mm}$ was placed under the electrode shirt. The human subject was sitting on chair with straight back without moving.

\section{MEAsurement Results}

All the measurements were done in the frequency range of $2 \mathrm{MHz}-20 \mathrm{MHz}$. The choice of the value of the lower level of the frequency is explained by the disappearance of the 
useful signal into the noise below $2 \mathrm{MHz}$. Moreover, the choice of the higher frequency range is related to the intention to use the device in noncontact measurement setup to the object and to cope with capacitive measurements. The chosen frequencies for the presentation in the figures are: $2 \mathrm{MHz}, 5 \mathrm{MHz}, 10 \mathrm{MHz}$ and $20 \mathrm{MHz}$.

In the cases of ES1-ES3, the reference waveforms of ECG and respiration were simultaneously gathered.

\section{A. Results of the Measurements by Using the DBR}

The results for evaluating the custom made measurement device and comparing the results in the case of human subject with the measurements that were done by using the DBR can be seen in Fig. 7.

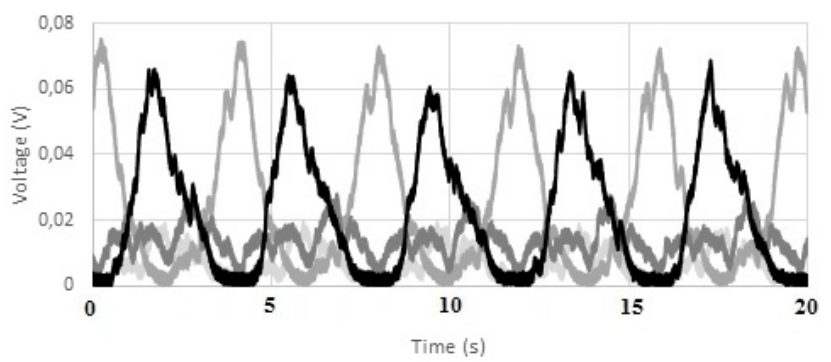

$\mathrm{F}=2 \mathrm{MHz}-\mathrm{F}=5 \mathrm{MHz} \quad \mathrm{F}=10 \mathrm{MHz} \quad-\mathrm{F}=20 \mathrm{MHz}$

Fig. 7. Results of the measurements by using the custom made measurement device in the case of DBR.

It can be seen that the HR appears most clearly in the case of $5 \mathrm{MHz}$ but is available also at $20 \mathrm{MHz}$. The RR is distinct at all of the chosen frequencies, showing the highest amplitudes at $5 \mathrm{MHz}$.

\section{B. Results of the Measurements on Human by Using the Electrodes in Direct Connection}

The measurements in the case of using the electrodes in direct contact to human body were carried out with the purpose of achieving the data for comparison with the results of capacitive measurements. The used EPC was: AB and CD (Fig. 3). The results can be seen in Fig. 8.

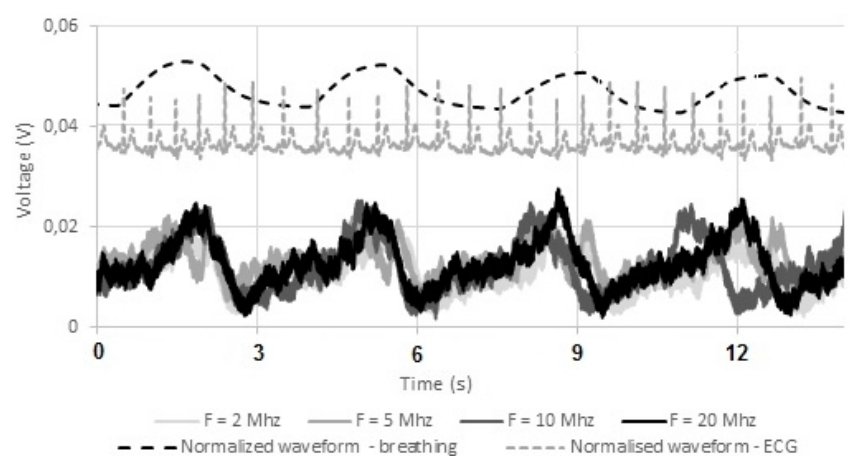

Fig. 8. Results of the measurements by using the custom made device in the case of using the electrodes in direct connection to human body.

The HR is lost into the high frequency noise, though, the $\mathrm{RR}$ is visible. The amplitude of RR shows similar value at all of the chosen excitation frequencies.

\section{Results of the Measurements by Using the Electrodes in} Capacitive Connection in the Case of ES1

With the intention of comparing the results of the measurements by using the custom made measurement device in different setups, the measurements were carried out by using two EPC's.

In the case of ES1, the following EPC's were used:

- EFIJGKHL and OP;

- NRPMQO and AEIDHL.

The first EPC sets the trunk vertically and second EPC horizontally between the electrodes (Fig. 4). The results can be seen in Fig. 9 and Fig. 10.

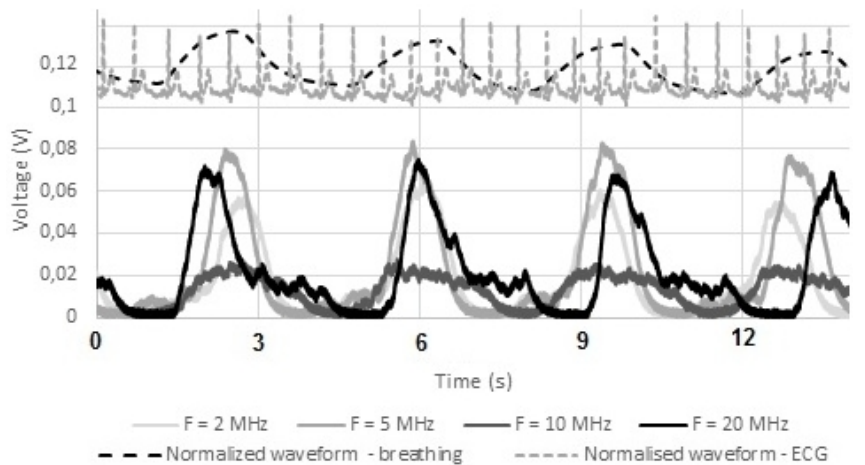

Fig. 9. Results of the measurements by using the custom made device in the case of EPC EFIJGKHL and OP of ES1.

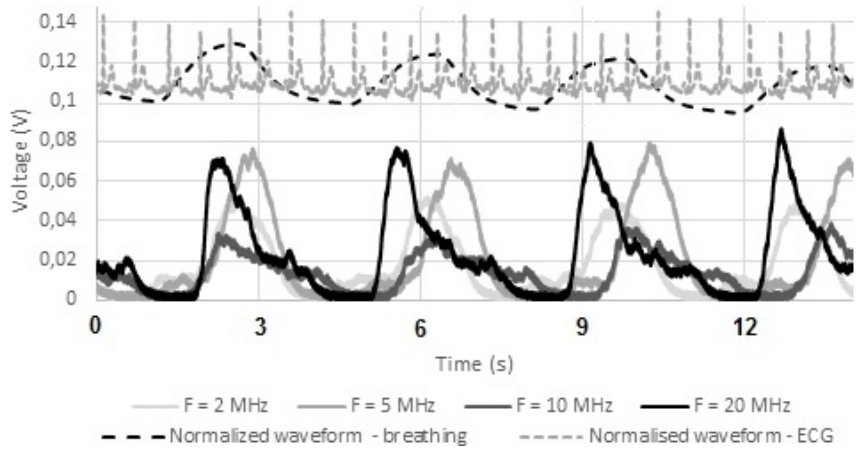

Fig. 10. Results of the measurements by using the custom made measurement device in the case of EPC NRPMQO and AEIDHL of ES1.

Concerning both figures (Fig. 9-Fig. 10), the HR can be assumed to be seen visually in the cases of two higher excitation frequencies, carried by the signal of RR. The RR is distinct at all of the chosen frequencies and in the cases of both experimented EPC's in the case of ES1.

D. Results of the Measurements by Using the Electrodes in Capacitive Connection in the Case of ES2

In the case of ES2, the following EPC's were used:

$-\mathrm{K}$ and $\mathrm{J}$;

$-\mathrm{E}$ and D.

EPC's of ES2 were orientated to find the access to HR. The result can see in Fig. 11 and Fig. 12.

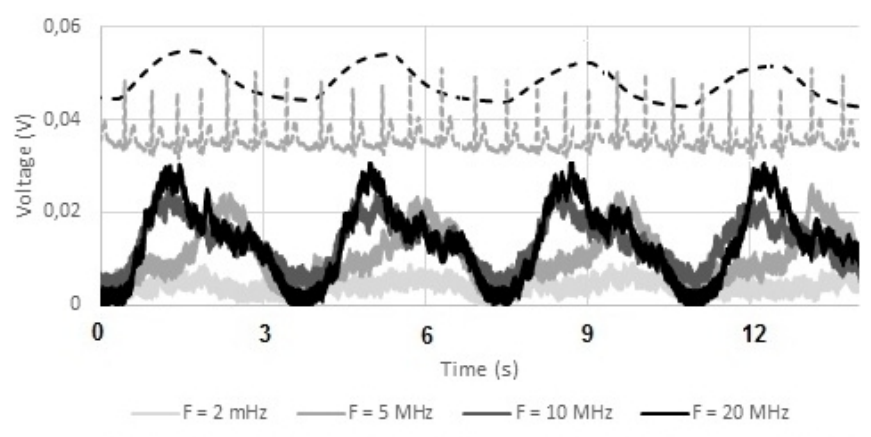

- - - Normalized waveform - breathing -----Normalised waveform-ECG Fig. 11. Results of the measurements by using the custom made measurement device in the case of EPC K and J of ES2. 


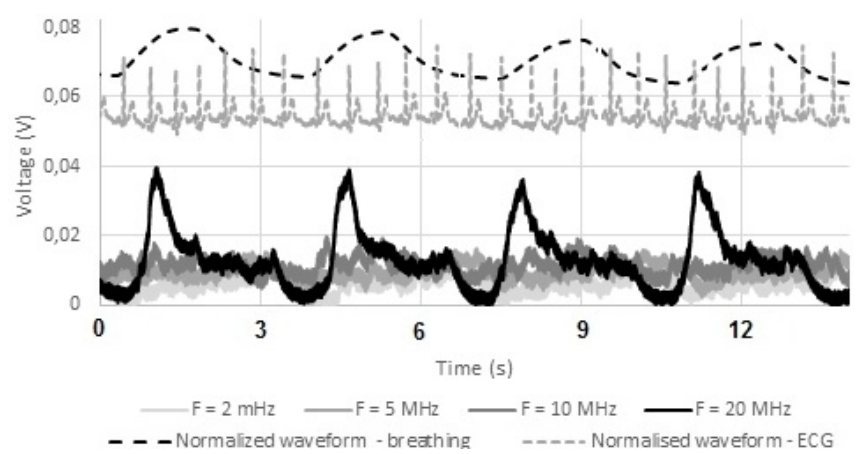

Fig. 12. Results of the measurements by using the custom made device in the case of EPC E and D of ES2.

It can be seen that the level of amplitude of RR increases with the increasing excitation frequency. The HR can't be visually fully recognized with certainty in any of the experimented EPC's in the case of ES2 because of the concurrent noise.

\section{E. Results of the Measurements by Using the Electrodes in Capacitive Connection in the Case of ES3}

Concerning ES3, the textile electrodes were connected to cover the trunk of human vertically. The only experimented EPC for ES3 was: ABCDIJ and EFGHKLMN. The results can be seen in Fig. 13.

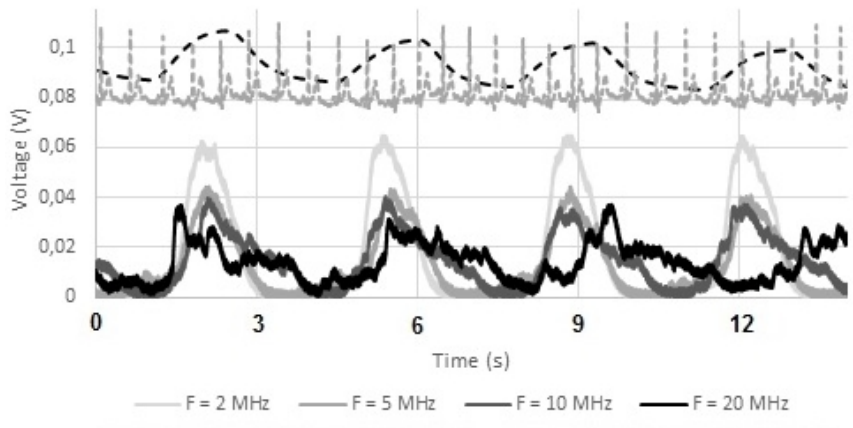
- - - Normalized waveform - breathing -- Normalised waveform - ECG

Fig. 13. Results of the measurements by using the custom made measurement device in the case of ES3.

In the case of Fig. 13, the amplitude of RR is the highest for the lowest chosen excitation frequency and the lowest for the highest chosen excitation frequency. The HR is visually most clearly available at the frequency of $20 \mathrm{MHz}$.

\section{DISCUSSION}

The frequency of the excitation signal has influence on the amplitude of RR in all the results. The excitation current finds a way through the mediums with lower impedance and at some point it may even not pervade the human body but travel through the skin surface. This means that the change of the voltage at the output of the device does not reflect the change of the impedance, caused by RR and HR, but the change of the cap between the capacitive electrodes and the skin surface. Anyhow, in the sense of the access to the RR and $\mathrm{HR}$, the requirement is the recognizable waveform at the output of the device, ready for the further analysis.

The amplitudes of the RR in the cases of contact electrodes and ES2 are showing the similar values. This reflects the impact of the size of the area of the electrodes. In the case of contact electrodes, the total area of contact surfaces is roughly $12.56 \mathrm{~cm}^{2}$ and in the case of $\mathrm{K}$ and $\mathrm{J}$ (ES2) $-96 \mathrm{~cm}^{2}$. The total area of the electrodes EFIJGKHL and OP in the case of ES1 is $1360 \mathrm{~cm}^{2}$.

For comparing the results of the measurements by using different EPC's and shirts at the same excitation frequency, the following graphs were created (Fig. 14-Fig. 17).

As the EPC's of ES2 were orientated to find the access to HR and the results of amplitudes of RR were two times lower than in the cases of ES1 and ES3, these results are not shown in the following graphs.

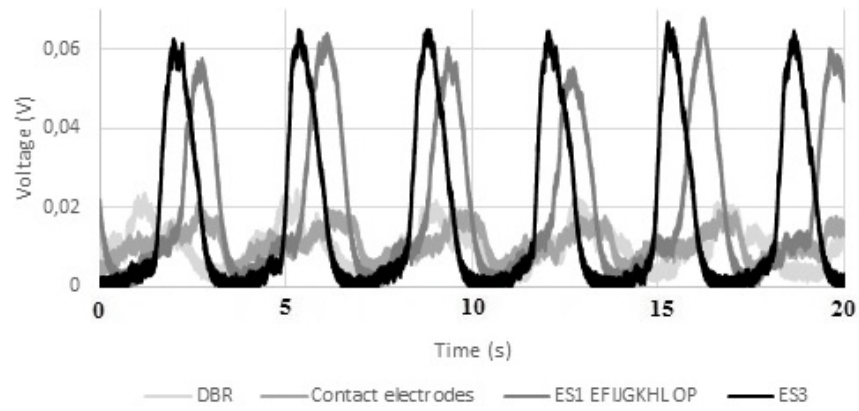

Fig. 14. Comparison of the results of the measurements by different electrodes and EPC's at the excitation frequency of $2 \mathrm{MHz}$.

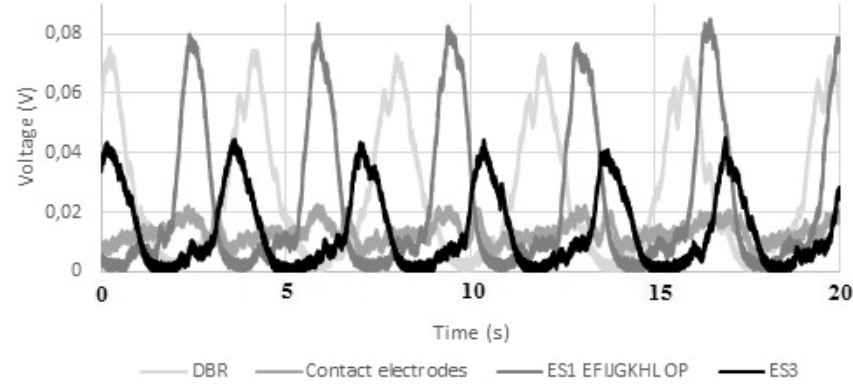

Fig. 15. Comparison of the results of the measurements by different electrodes and EPC's at the excitation frequency of $5 \mathrm{MHz}$

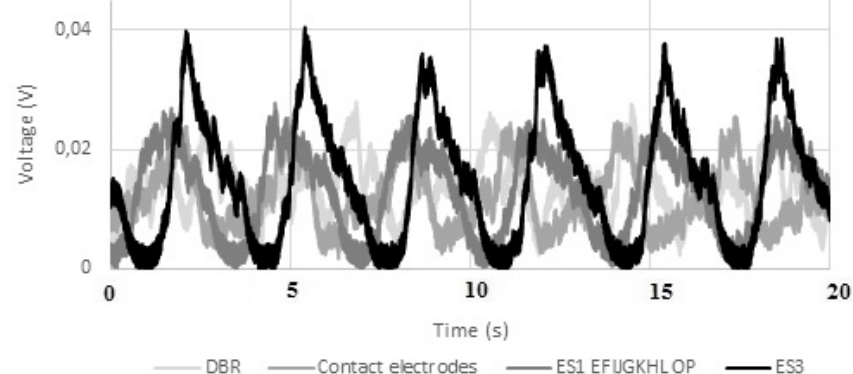

Fig. 16. Comparison of the results of the measurements by different electrodes and EPC's at the excitation frequency of $10 \mathrm{MHz}$.

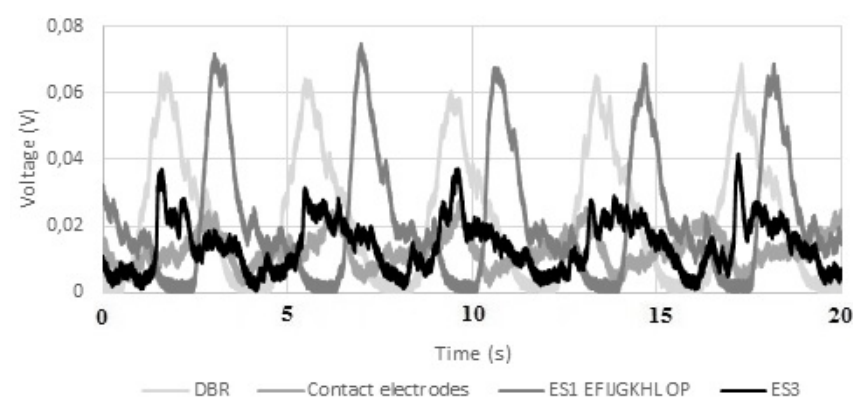

Fig. 17. Comparison of the results of the measurements by different electrodes and EPC's at the excitation frequency of $20 \mathrm{MHz}$.

The peak values of the amplitudes of the measured signals at chosen frequencies, caused by RR, can be seen in Table I. 
TABLE I. PEAK VALUES OF THE AMPLITUDES OF THE SIGNALS, CAUSED BY RR, SHOWN IN FIG. 14-FIG. 17.

\begin{tabular}{|c|c|c|c|c|}
\hline Item & $\begin{array}{c}\text { Amplitude } \\
\text { of RR in } \\
\text { the case of } \\
\mathbf{F}=\mathbf{2} \mathbf{~ M H z} \\
\mathbf{( m V )}\end{array}$ & $\begin{array}{c}\text { Amplitude } \\
\text { of RR in } \\
\text { the case of } \\
\mathbf{F}=\mathbf{5} \mathbf{~ M H z} \\
(\mathbf{m V})\end{array}$ & $\begin{array}{c}\text { Amplitude } \\
\text { of RR in } \\
\text { the case of } \\
\mathbf{F}=\mathbf{1 0} \\
\mathbf{M H z} \mathbf{( m V )}\end{array}$ & $\begin{array}{c}\text { Amplitude } \\
\text { of RR in } \\
\text { the case of } \\
\mathbf{F}=\mathbf{2 0} \\
\mathbf{M H z} \mathbf{( m V})\end{array}$ \\
\hline DBR & 24.7 & 72.8 & 27.1 & 67.9 \\
\hline $\begin{array}{c}\text { Contact } \\
\text { electrodes }\end{array}$ & 20.4 & 22 & 24.4 & 23.3 \\
\hline $\begin{array}{c}\text { ES1 } \\
\text { EFIJKH } \\
\text { LOP }\end{array}$ & 64.9 & 84.2 & 27.8 & 74.5 \\
\hline ES3 & 65.3 & 44.2 & 40.1 & 40.8 \\
\hline
\end{tabular}

The RR is showing the highest amplitude in the case of ES1 at the frequency of $5 \mathrm{MHz}$ - reaching up to $80 \mathrm{mV}$. The best result in the case of ES3 is achieved at the frequency of $2 \mathrm{MHz}$, achieving the amplitude of more than $60 \mathrm{mV}$. This is illustrating the effectiveness of larger surface area of the electrodes.

In the case of $10 \mathrm{MHz}$, the amplitude of the signal of RR in the case of chosen EPC of ES1 is two times lower than in other frequencies. This can be explained by the influence of the parasitic capacitances and inductances, caused for example by the length of the wires [13].

Concerning HR, the best results have been achieved by using ES3 in the case of $20 \mathrm{MHz}$ (Fig. 18) - the amplitude of the change reaches about $10 \mathrm{mV}$. The typical parameters of HR and RR are 60-100 heart beats per minute and 12-18 breaths per minute respectively. This makes about 5 heart beats per one breathing cycle (depending on different factors). This ratio can be seen also in Fig. 18.

The waveform that can be seen Fig. 18, is measured at the output of custom made impedance measurement device is filtered by using a Bessel bandpass filter in the frequency band of $10 \mathrm{mHz}-2 \mathrm{~Hz}$. The result is shown together with synchronously gathered ECG. The result and the reference waveform of ECG are normalised in y-axis.

The results of the measurements by using the custom made device contain the high frequency noise, blurring the graphs. The low pass filtering should be done as close to the oscilloscope input as possible to diminish the noise.

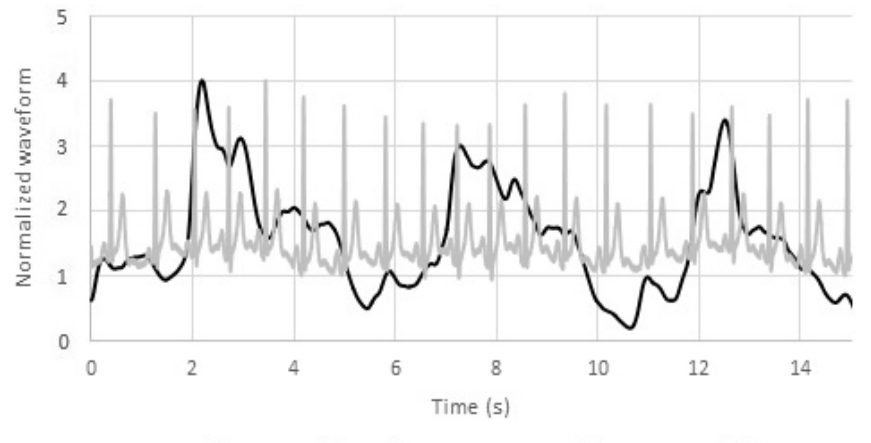

Fig. 18. Result of the measurement by using the custom made measurement device in the case of ES3 at the frequency of $20 \mathrm{MHz}$ and the reference ECG in normalized scale (y-axis).

The spectrum of the signal, presented in Fig. 18, can be seen in Fig. 19, where the distribution of the energy in frequency axis is available. The fundamental frequency of respiration is clearly available. Nevertheless, due to the high frequency noise, HR is not surely identifiable. It can be expected that in the case of longer measurement data sets (current sets are of the length of 5 breathing cycles) the result concerning HR would give visible result.

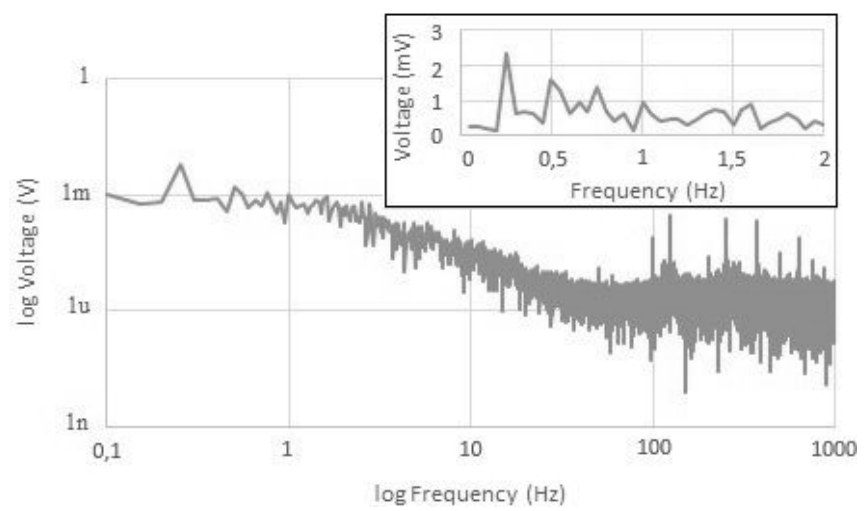

Fig. 19. Spectrum of the signal, measured by custom made impedance measurement device in the case of ES3 at the excitation frequency of $20 \mathrm{MHz}$ (the smaller graph in the frame shows the same spectrum in narrower linear scale)

For comparison with the results of a commercial impedance measurement device, reference measurement was done by using HF2IS impedance spectroscope. The results in the case of ES3 at the excitation frequency of $20 \mathrm{MHz}$ can be seen in Fig. 20. In the graph, only the trends of the normalized signals are presented while the ratios of the signal remain the same. The DC offsets are shifted during the signal processing for better visual representation.

In the case of HF2IS impedance spectroscope, the RR is available at all of the excitation frequencies. The amplitudes of the signals are of similar value with slightly better result in the case of ES3 - the change may come from the displacement of the electrode shirt during the measurement.

$\mathrm{HR}$ is visually available in the case of using the DBR and apparent also in the case of ES1. In fact, concerning Fig. 19, the HR is available in all of the cases if the signal is magnified, carried by the RR - discussed already in [12].

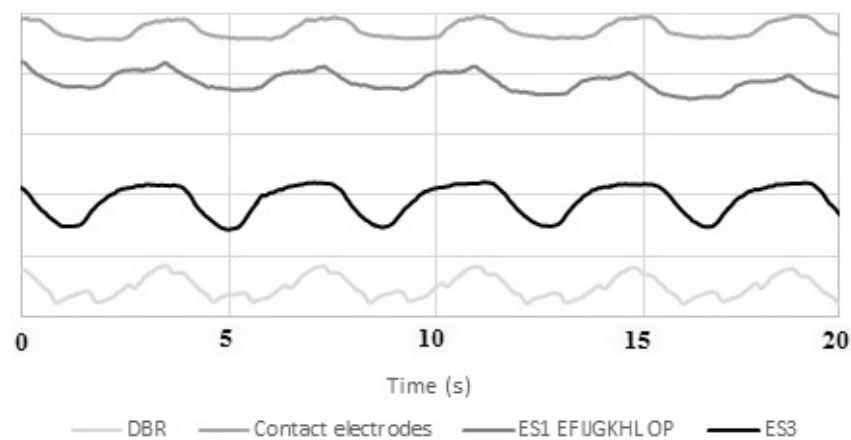

Fig. 20. Trends of the normalized signals, measured by HF2IS impedance spectroscope in the case of ES3 at the excitation frequency of $20 \mathrm{MHz}$.

The change of the amplitude of measured signal, caused by the movements of the subject's body during the measurement experiments, is distracting the result for visual interpretation. Nevertheless, in the case of periodical movements like imitating swimming or lifting the shoulders, the RR can still be recognized. Concerning the motion artifact, the help of the elaborate design in the form of tight clothing and special algorithms should be developed. 


\section{CONCLUSIONS}

The prototype of an impedance measurement device has been implemented and number of electrode shirts prepared to evaluate the properties and the behaviour of the device. The measurements on human subject have been done using the direct contact and capacitive electrodes of different types in various EPC's to assess the availability of RR and HR. The influence of motion artifact is not considered, though, the occasional study of hand movements during the experiments showed the vast effect.

The results of the measurements showed the visible availability of RR in all of the experiment setups at all of the chosen excitation frequencies. HR is visually available, typically in the cases of highest chosen excitation frequency: $20 \mathrm{MHz}$. HR is interfered by the high frequency noise.

The results are promising, exhibiting the usability of the proposed idea and realization of the measurement device.

The future work includes the redesign of the blocks 6-7 to suit for incorporation into an outer case. The usage of sine wave as excitation signal and the switching onto battery power will be researched. The plans include also the development of next generation of electrode shirt according to the previously published [13] and the current results, with the attention to the influence and reduction of the motion artifacts. The final outcome is presumed to be constituted as a wearable device for monitoring the RR and HR.

\section{REFERENCES}

[1] L. Guo, L. Berglin, U. Wiklund, H. Mattila, "Design of a garmentbased sensing system for breathing monitoring", in Textile Research J., vol. 83, no. 5, pp. 499-509, 2012. [Online]. Available: http://journals.sagepub.com/doi/pdf/10.1177/0040517512444336

[2] P. Sanchez, C. R. Zamarreno, M. Hernaez, I. R. Matias, F. J. Arregui, "Exhaled breath optical fiber sensor based on LMRs for respiration monitoring", in IEEE Sensors, 2014. [Online]. Available: https://doi.org/10.1109/ICSENS.2014.6985209

[3] A. Richer, A. Adler, "Eddy current based flexible sensor for contactless measurement of breathing", in Proc. IEEE IMTC 2005 Instr. Meas., Ottawa, Canada, 2015 pp. 257-260. [Online]. Available: https://doi.org/10.1109/IMTC.2005.1604112

[4] T. Vuorela, J. Vanhala, V.-P. Seppa, J. Hyttinen, "Two portable long term measurement devices for ECG and bioimpedance", in Proc. $2^{\text {nd }}$ IEEE Int. ICST Conf. on Pervasive Computing Technologies for Healthcare, Tampere, Finland, 2008, pp. 159-162. [Online]. Available: https://doi.org/10.1109/PCTHEALTH.2008.4571061

[5] J. Wtorek, A. Bujnowski, M. Lewandowska, J. Ruminski, M. Kaczmarek, "Simultaneous monitoring of heart performance and respiration activity", in Proc. 3rd Conf. on Human System Interactions (HIS), 2010, pp. 661-665. [Online]. Available: https://doi.org/10.1109/HSI.2010.5514499

[6] S. Hong, J. Lee, H.-J. Yoo, "Wearable lung-health monitoring system with electrical impedance tomography", in Proc. Annual Int. Conf. 37th IEEE Eng. Med. Bio. Soc. (EMBC), 2015, pp. 1707-1710. [Online]. Available: https://doi.org/10.1109/EMBC.2015.7318706

[7] S. Grimnes, O. G. Martinsen, Bioimpedance \& Bioelectricity Basics. London, Great Britain: Academic, 2008. p. 201.

[8] M. Rist, M. Min, "Dynamic reference for evaluation of bioimpedance spectroscopy devices", in Proc. IEEE $16^{\text {th }}$ Biennial Baltic Electronics Conf. (BEC 2016), Tallinn, Estonia, 2016, pp. 107-110. [Online]. Available: https://doi.org/10.1109/BEC.2016.7743740

[9] HF2 User Manual - LabOne Edition, Zurich Instruments, 2016. [Online] Available: http://www.zhinst.com/manuals/hf2

[10] M. Metshein, T. Parve, "Electrical bioimpedance based monitoring of cardiac and respiratory activity: positioning of capacitive electrodes for wearable human monitoring device", in Int. J. of Bioelectromagnetism, vol. 17, no. 2, pp 64-74, 2015. [Online] Available: http://www.ijbem.org/volume17/number2/ijbem_vol17_ no2_pp64-74.pdf

[11] D. P. Bernstein, "A new stroke volume equation for thoracic electrical bioimpedance: theory and rationale", in Crit. Care Med., vol. 14, pp. 904-909, 1986. [Online]. Available: https://doi.org/10.1097/ 00003246-198610000-00017

[12] M. Metshein, "A device for measuring the electrical bioimpedance with variety of electrode placements for monitoring the breathing and heart rate", in Proc. IEEE 26th Irish Signals and Systems Conf. (ISSC), Carlow, Ireland, 2015, pp. 1-4. [Online]. Available: https://doi.org/10.1109/ISSC.2015.7163748

[13] M. Metshein, R. Gordon, "On the possibility of detecting the electrical bioimpedance of human body by using non-contact electrodes in capacitive connection", in Proc. IEEE $16^{\text {th }}$ Biennial Baltic Electronics Conf. (BEC 2016), Tallinn, Estonia, 2016, pp. 171-174. [Online]. Available: https://doi.org/10.1109/BEC.2016. 7743756 